\title{
Dirichlet Average of Generalized Miller-Ross Function and Fractional Derivative
}

\author{
Mohd. Farman Ali ${ }^{1}$, Manoj Sharma ${ }^{2}$, Lakshmi Narayan Mishra ${ }^{3,4}$, Vishnu N. Mishra ${ }^{5 *}$ \\ ${ }^{1}$ School of Mathematics and Allied Sciences, Jiwaji University, Gwalior \\ ${ }^{2}$ Department of Mathematics RJIT, BSF Academy, Tekanpur, Gwalior \\ ${ }^{3}$ Department of Mathematics, National Institute of Technology, Silchar - 788 010, District - Cachar (Assam), India \\ ${ }^{4}$ L. 1627 Awadh Puri Colony Beniganj, Phase - III, Opposite - Industrial Training Institute (I.T.I.), Faizabad - 224001 (Uttar Pradesh), \\ India \\ ${ }^{5}$ Applied Mathematics and Humanities Department, Sardar Vallabhbhai National Institute of Technology, Ichchhanath Mahadev \\ Dumas Road, Surat - 395007 (Gujarat), India \\ *Corresponding author: vishnunarayanmishra@gmail.com
}

Received December 09, 2014; Revised January 14, 2015; Accepted March 01, 2015

\begin{abstract}
The object of the present paper is to establish the results of single Dirichlet average of Generalized Miller-Ross Function, using Riemann-Liouville Fractional Integral. The Generalized Miller-Ross Function can be measured as a Dirichlet average and connected with fractional calculus. In this paper the solution comes in compact form of single Dirichlet average of Generalized Miller-Ross Function. The special cases of our results are same as earlier obtained by Saxena et al. [12], for single Dirichlet average of Generalized Miller-Ross Function.
\end{abstract}

Keywords: Dirichlet average, Generalized Miller-Ross Function, fractional derivative and Fractional calculus operators

Cite This Article: Mohd. Farman Ali, Manoj Sharma, Lakshmi Narayan Mishra, and Vishnu N. Mishra, "Dirichlet Average of Generalized Miller-Ross Function and Fractional Derivative." Turkish Journal of Analysis and Number Theory, vol. 3, no. 1 (2015): 30-32. doi: 10.12691/tjant-3-1-7.

\section{Introduction}

Carlson [1-5] has defined Dirichlet average of functions which represents certain type of integral average with respect to Dirichlet measure. He showed that various important special functions can be derived as Dirichlet averages for the ordinary simple functions like $x^{t}, e^{x}$ etc. He has also pointed out [3] that the hidden symmetry of all special functions which provided their various transformations can be obtained by averaging $x^{n}, e^{x}$ etc. Thus he established a unique process towards the unification of special functions by averaging a limited number of ordinary functions. Almost all known special functions and their well known properties have been derived by this process.

Recently, Gupta and Agarwal [9,10] found that averaging process is not altogether new but directly connected with the old theory of fractional derivative. Carlson overlooked this connection whereas he has applied fractional derivative in so many cases during his entire work. Deora and Banerji [6] have found the double Dirichlet average of $\mathrm{e}^{\mathrm{x}}$ by using fractional derivatives and they have also found the Triple Dirichlet Average of $x^{t}$ by using fractional derivatives [7].

In the present paper the Dirichlet average of Generalized Miller-Ross Function has been obtained.

\section{Definitions}

Some definitions which are necessary in the preparation of this paper.

\subsection{Standard Simplex in $R^{n}, n \geq 1$ :}

Denote the standard simplex in $R^{n}, n \geq 1$ by [[1], p. $62]$.

$$
\begin{aligned}
& E=E_{n}=\left\{S\left(u_{1}, u_{2}, \ldots u_{n}\right):\right. \\
& \left.u_{1} \geq 0, \ldots u_{n} \geq 0, u_{1}+u_{2}+\ldots+u_{n} \leq 1\right\}
\end{aligned}
$$

\subsection{Dirichlet Measure}

Let $b \in C^{k}, k \geq 2$ and let $E=E_{k-1}$ be the standard simplex in $R^{k-1}$ The complex measure $\mu_{b}$ is defined by E [1].

$$
\begin{aligned}
& d \mu_{b}(u)=\frac{1}{B(b)} u_{1}^{b_{1}-1} \ldots u_{k-1}^{b_{k-1}-1}\left(1-u_{1}-\ldots-u_{k-1}\right) \\
& b_{k}^{-1} d u_{1} \ldots d u_{k-1}
\end{aligned}
$$

known as Dirichlet measure.

Here 


$$
\begin{aligned}
& B(b)=B(b 1, \ldots b k)=\frac{\Gamma\left(b_{1}\right) \ldots \Gamma\left(b_{k}\right)}{\Gamma\left(b_{1}+\ldots+b_{k}\right)}, \\
& C_{>}=\{z \in z: z \neq 0,|p h z|<\pi / 2\},
\end{aligned}
$$

open right half plane and $C_{>} \mathrm{k}$ is the $k^{\text {th }}$ Cartesian power of $C_{>}$.

\subsection{Dirichlet Average[[1], p. 75]}

Let $\Omega$ be the convex set in $C_{>}$, let $z=\left(z_{1}, \ldots, z_{k}\right) \in \Omega^{\mathrm{k}}, \mathrm{k} \geq 2$ and let $u . z$ be a convex combination of $z_{1}, \ldots, z_{k}$. Let $f$ be a measureable function on $\Omega$ and let $\mu_{b}$ be a Dirichlet measure on the standard simplex $E$ in $R^{k-1}$.Define

$$
F(b, z)=\int_{E} f(u . z) d \mu_{b}(u)
$$

$\mathrm{F}$ is the Dirichlet measure of $f$ with variables $z=\left(z_{1}, \ldots, z_{k}\right)$ and parameters $b=\left(b_{1}, \ldots b_{k}\right)$.

Here

$$
u . z=\sum_{i=1}^{k} u_{i} z_{i} \text { and } u_{k}=1-u_{1}-\ldots-u_{k-1}
$$

If $k=1$, define $F(b, z)=f(z)$

\subsection{Generalized Miller-Ross Function}

This function is introduced by the author as follows:

$$
\begin{aligned}
& { }_{0}^{a} N_{p, q}^{\alpha, \beta}\left(a_{1} \ldots a_{p} ;, b_{1} \ldots b_{q} ; x^{0}\right)={ }_{0}^{a} N_{p, q}^{\alpha, \beta}(x) \\
& =\sum_{n=o}^{\infty} \frac{\left(a_{1}\right)_{n} \ldots\left(a_{p}\right)_{n}}{\left(b_{1}\right)_{n} \ldots\left(b_{q}\right)_{n}} \frac{a^{n} x^{n+\beta}}{\Gamma(\alpha n+\beta+1)} .
\end{aligned}
$$

Here, $p$ upper parameters $a_{1}, a_{2}, \ldots a_{p}$ and $q$ lower parameters $b_{1}, b_{2}, \ldots b_{q}, \alpha, \beta \epsilon C, R(\alpha)>0, R(\beta)>0 \quad$ and $\left(a_{j}\right)_{k}\left(b_{j}\right)_{k}$ are pochammer symbols. The function (3.6.1.1) is defined when none of the denominator parameters $b_{j} s, j=1,2, \ldots q$ is a negative integer or zero. If any parameter $a_{j}$ is negative then the function (2.4) terminates into a polynomial in $\mathrm{x}$. By using ratio test, it is evident that function (2.4) is convergent for all $\mathrm{x}$, when $q \geq p$, it is convergent for $|x|<1$ when $p=q+1$, divergent when $p>q+1$. In some cases the series is convergent for $x=1, x=-1$. Let us consider take,

$$
\beta=\sum_{j=1}^{p} a_{j}-\sum_{j=1}^{q} b_{j}
$$

when $p=q+1$, the series is absolutely convergent for $|x|=1 \quad$ if $\quad R(\beta)<0$, convergent for $x=-1$ if $0 \leq R(\beta)<1$ and divergent for $|x|=1$ if $1 \leq R(\beta)$ which is a special case of Wright function.

\subsection{Fractional Derivative [[8], p. 181]}

The theory of fractional derivative with respect to an arbitrary function has been used by Erdelyi [8]. The general definition for the fractional derivative of order $\alpha$ found in the literature on the "Riemann-Liouville integral" is

$$
D_{z}^{\alpha} F(z)=\frac{1}{\Gamma(-\alpha)} \int_{0}^{z} F(t)(z-t)^{-\alpha-1} d t
$$

where $\operatorname{Re}(\alpha)<0$ and $F(x)$ is the form of $x^{p} f(x)$, where $f(x)$ is analytic at $x=0$

\section{Equivalence}

In this section we shall prove the equivalence of single Dirichlet average of Generalized Miller-Ross Function $(k=2)$ with the fractional derivative i.e.

$$
\begin{aligned}
& S\left(\beta, \beta^{\prime}, x, y\right)=\frac{\Gamma\left(\beta+\beta^{\prime}\right)}{\Gamma \beta}(x-y)^{1-\beta-\beta^{\prime}} \\
& D_{x-y}^{-\beta^{\prime}}{ }_{0}^{a} N_{p, q}^{\alpha, \beta}(x)(x-y)^{\beta-1} .
\end{aligned}
$$

\section{Proof:}

$$
\begin{aligned}
& S\left(\beta, \beta^{\prime}, x, y\right)=\sum_{n=o}^{\infty} \frac{\left(a_{1}\right)_{n} \ldots\left(a_{p}\right)_{n}}{\left(b_{1}\right)_{n} \ldots\left(b_{q}\right)_{n}} \frac{a^{n}}{\Gamma(\alpha n+\beta+1)} \\
& R_{n}\left(\beta, \beta^{\prime}, x, y\right) \\
& =\sum_{n=o}^{\infty} \frac{\left(a_{1}\right)_{n} \ldots\left(a_{p}\right)_{n}}{\left(b_{1}\right)_{n} \ldots\left(b_{q}\right)_{n}} \frac{a^{n}}{\Gamma(\alpha n+\beta+1)} \frac{\Gamma\left(\beta+\beta^{\prime}\right)}{\Gamma \beta \Gamma \beta^{\prime}} \\
& \int_{0}^{1}[u x+(1-u) y]^{n+\beta} u^{\beta-1}(1-u)^{\beta^{\prime}-1} d u
\end{aligned}
$$

Putting $u(x-y)=t$, we have

$$
\begin{aligned}
& =\sum_{n=o}^{\infty} \frac{\left(a_{1}\right)_{n} \ldots\left(a_{p}\right)_{n}}{\left(b_{1}\right)_{n} \ldots\left(b_{q}\right)_{n}} \frac{a^{n}}{\Gamma(\alpha n+\beta+1)} \frac{\Gamma\left(\beta+\beta^{\prime}\right)}{\Gamma \beta \Gamma \beta^{\prime}} \\
& \int_{0}^{x-y}[t+y]^{n+\beta}\left(\frac{t}{x-y}\right)^{\beta-1}\left(1-\frac{t}{x-y}\right)^{\beta^{\prime}-1} \frac{d t}{x-y} .
\end{aligned}
$$

On changing the order of integration and summation, we have

$$
\begin{aligned}
& =(x-y)^{1-\beta-\beta^{\prime}} \frac{\Gamma\left(\beta+\beta^{\prime}\right)}{\Gamma \beta \Gamma \beta^{\prime}} \\
& \int_{0}^{x-y} \sum_{n=o}^{\infty} \frac{\left(a_{1}\right)_{n} \ldots\left(a_{p}\right)_{n}}{\left(b_{1}\right)_{n} \ldots\left(b_{q}\right)_{n}} \frac{a^{n}}{\Gamma(\alpha n+\beta+1)} \\
& {[t+y]^{n+\beta}(t)^{\beta-1}(x-y-t)^{\beta^{\prime}-1} d t}
\end{aligned}
$$

$\mathrm{Or}$ 


$$
\begin{aligned}
& =(x-y)^{1-\beta-\beta^{\prime}} \frac{\Gamma\left(\beta+\beta^{\prime}\right)}{\Gamma \beta \Gamma \beta^{\prime}} \\
& \int_{0}^{x-y}{ }_{0}^{a} N_{p, q}^{\alpha, \beta}(y+t)(t)^{\beta-1}(x-y-t)^{\beta^{\prime}-1} d t .
\end{aligned}
$$

Hence by the definition of fractional derivative, we get

$$
\begin{aligned}
& S\left(\beta, \beta^{\prime}, x, y\right)=(x-y)^{1-\beta-\beta^{\prime}} \\
& \frac{\Gamma\left(\beta+\beta^{\prime}\right)}{\Gamma \beta} D_{x-y}^{-\beta_{0}^{\prime}}{ }_{0}^{a} N_{p, q}^{\alpha, \beta}(x)(x-y)^{\beta-1} .
\end{aligned}
$$

This completes the analysis.

\section{Particular Cases}

If $\beta^{\prime}=\gamma-\beta, \beta=0, y=0, \alpha=1, a=1$ and no upper and lower parameter in (3.1) then

$$
\begin{aligned}
& S(0, \gamma-\beta, x, 0)=(x)^{1-\gamma} \frac{\Gamma \gamma}{\Gamma \beta} D_{x}^{-\gamma} e^{x}(x)^{\beta-1} \\
& ={ }_{1} F_{1}(\beta ; \gamma ; x) .
\end{aligned}
$$

This confluent hyper geometric function [11]

$$
S(\beta, \gamma-\beta, x, 0)=\frac{1}{2 \pi i} \int_{c-i \infty}^{c+i \infty} \Gamma s \frac{\Gamma(\beta-s)}{\Gamma(\gamma-s)}(-x)^{-s} d s .
$$

Then

$$
S(\beta, \gamma-\beta, x, 0)=\frac{\Gamma(\gamma)}{\Gamma(\beta)}\left[H_{1,2}^{1,1}\left(\begin{array}{c}
\left(1-x \mid \begin{array}{c}
(1) \\
(0,1),(1-\gamma, 1)
\end{array}\right)
\end{array}\right]\right.
$$

(ii) If $\beta^{\prime}=\xi-\beta$ and from (4.1), then

$$
\begin{aligned}
& S(\beta, \xi-\beta ; x, 0)=\frac{\Gamma(\xi)}{\Gamma(\beta)} x^{1-\xi} D_{x}^{\beta-\xi} e^{x} x^{\beta-1} \\
& S(\beta, \xi-\beta ; x, 0)={ }_{1} F_{1}(\beta ; \xi ; x)=\Gamma(\xi) E_{1, \xi}^{\beta}(x)
\end{aligned}
$$

where $E_{1, \xi}^{\beta}(x)$ be the generalization of Mittag-Leffler function [12].

\section{Applications}

Dirichlet average is average given by Dirichlet. The Dirichlet average of elementary function like power function, exponential function etc is given by many notable mathematician, Actually, We have convert the elementary function into the summation form after that taking Dirichlet average of those function, using fractional integral and get new results. These results will be used in future by mathematician and scientist. Thus we have find a connection Dirichlet average of a function and fractional integral.

\section{Acknowledgements}

The authors express their thanks to the anonymous learned referee(s) and the editor for their constructive comments, valuable suggestions, which resulted in the subsequent improvement of this research article. The authors are grateful to all editorial board members and reviewers of esteemed journal i.e. Turkish Journal of Applied Analysis and Number Theory (TJANT). All the authors conceived of the study and participated in its design and coordination. All authors drafted the manuscript, participated in the sequence alignment. All the authors read and approved the final version of manuscript. The authors declare that there is no conflict of interests regarding the publication of this research article.

\section{References}

[1] Carlson, B.C., Special Function of Applied Mathematics, Academic Press, New York, 1977.

[2] Carlson, B.C., Appell's function $F_{4}$ as a double average, SIAM J.Math. Anal. 6 (1975), 960-965.

[3] Carlson, B.C., Hidden symmetries of special functions, SIAM Rev. 12 (1970), 332-345.

[4] Carlson, B.C., Dirichlet averages of $x^{t} \log x$, SIAM J.Math. Anal. 18(2) (1987), 550-565.

[5] Carlson, B.C., A connection between elementary functions and higher transcendental functions, SIAM J. Appl. Math. 17 (1969), 116-140.

[6] Deora, Y. and Banerji, P.K., Double Dirichlet average of ex using fractional derivatives, J. Fractional Calculus 3 (1993), 81-86.

[7] Deora, Y. and Banerji, P.K., Double Dirichlet average and fractional derivatives, Rev.Tec.Ing.Univ. Zulia 16 (2) (1993), 157161.

[8] Erdelyi, A., Magnus, W., Oberhettinger, F. and Tricomi , F.G., Tables of Integral Transforms, Vol. 2 McGraw-Hill, New York, 1954.

[9] Gupta,S.C. and Agrawal, B.M., Dirichlet average and fractional derivatives, J. Indian Acad.Math. 12(1) (1990), 103-115.

[10] Gupta,S.C. and Agrawal, Double Dirichlet average of ex using fractional derivatives, Ganita Sandesh 5 (1) (1991),47-52.

[11] Mathai, A.M. and Saxena,R.K., The H-Function with Applications in Stastistics and other Disciplines, Wiley Halsted, New York, 1978.

[12] Saxena,R.K., Mathai,A.M and Haubold, H.J., Unified fractional kinetic equation and a fractional diffusion equation, $\mathrm{J}$. Astrophysics and Space Science 209 (2004), 299-310.

[13] Sharma, Manoj and Jain, Renu, Dirichlet Average and Fractional Derivatie, J. Indian Acad. Math. Vol. 12, No. 1(1990). 